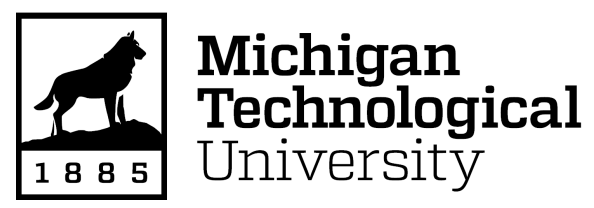

Michigan Technological University Digital Commons @ Michigan Tech

Michigan Tech Publications

3-21-2018

\title{
Spatial dispersion of index components required for building invisibility cloak medium from photonic crystals
}

\author{
Saeid Jamilan \\ Michigan Technological University, sjamilan@mtu.edu \\ George Semouchkin \\ Michigan Technological University, gbsemouc@mtu.edu \\ Navid Gandji \\ Michigan Technological University \\ Elena Semouchkina \\ Michigan Technological University, esemouch@mtu.edu
}

Follow this and additional works at: https://digitalcommons.mtu.edu/michigantech-p

Part of the Computer Sciences Commons, and the Electrical and Computer Engineering Commons

\section{Recommended Citation}

Jamilan, S., Semouchkin, G., Gandji, N., \& Semouchkina, E. (2018). Spatial dispersion of index components required for building invisibility cloak medium from photonic crystals. Journal of Optics, 20(4).

http://dx.doi.org/10.1088/2040-8986/aab25c

Retrieved from: https://digitalcommons.mtu.edu/michigantech-p/654

Follow this and additional works at: https://digitalcommons.mtu.edu/michigantech-p

Part of the Computer Sciences Commons, and the Electrical and Computer Engineering Commons 
PAPER

\section{Spatial dispersion of index components required for building invisibility cloak medium from photonic crystals}

To cite this article: Saeid Jamilan et al 2018 J. Opt. 20045102

\section{Manuscript version: Accepted Manuscript}

Accepted Manuscript is "the version of the article accepted for publication including all changes made as a result of the peer review process, and which may also include the addition to the article by IOP Publishing of a header, an article ID, a cover sheet and/or an 'Accepted

Manuscript' watermark, but excluding any other editing, typesetting or other changes made by IOP Publishing and/or its licensors"

This Accepted Manuscript is (c) (c) 2018 IOP Publishing Ltd.

During the embargo period (the 12 month period from the publication of the Version of Record of this article), the Accepted Manuscript is fully protected by copyright and cannot be reused or reposted elsewhere.

As the Version of Record of this article is going to be / has been published on a subscription basis, this Accepted Manuscript is available for reuse under a CC BY-NC-ND 3.0 licence after the 12 month embargo period.

After the embargo period, everyone is permitted to use copy and redistribute this article for non-commercial purposes only, provided that they adhere to all the terms of the licence https://creativecommons.org/licences/by-nc-nd/3.0

Although reasonable endeavours have been taken to obtain all necessary permissions from third parties to include their copyrighted content within this article, their full citation and copyright line may not be present in this Accepted Manuscript version. Before using any content from this article, please refer to the Version of Record on IOPscience once published for full citation and copyright details, as permissions will likely be required. All third party content is fully copyright protected, unless specifically stated otherwise in the figure caption in the Version of Record.

View the article online for updates and enhancements. 


\title{
Spatial dispersion of index components required for building invisibility cloak medium from photonic crystals
}

Department of Electrical and Computer Engineering, Michigan Technological University, 1400 Townsend Drive, Houghton, MI 49931, USA.

E-mail: sjamilan@mtu.edu

\begin{abstract}
The opportunities to use dielectric Photonic Crystals (PhCs) as the media of cylindrical invisibility cloaks, designed using Transformation Optics (TO) concepts, are investigated. It is shown that TO-based prescriptions for radial index dispersion, responsible for turning waves around hidden objects, can be dropped, if $\mathrm{PhC}$ media support selfcollimation of waves in bent crystals. Otherwise, to provide prescribed anisotropy of index dispersion, it is possible to employ PhCs with rectangular lattices. It is found, however, that at acceptable cloak thicknesses, modifications of crystal parameters do not allow for achieving prescribed level of index anisotropy. This problem is solved by finding reduced spatial dispersion law for radial index component, which is characterized by decreased against TOprescriptions values near the target and increased values in outer layers of the cloak. The cloak utilizing reduced prescriptions for indices is shown to perform almost as efficient, as TO-based cloak, in terms of both wave front restoration behind the target and reducing total scattering cross-width of the target.
\end{abstract}

Keywords: transformation optics, invisibility cloak, photonic crystals, dielectric rod array.

\section{Introduction}

Transformation optics (TO) has opened new perspectives for designing advanced electromagnetic (EM) devices with superior functionalities [1]. TO employs coordinate transformations to control EM wave paths by imposing specific spatial dispersion of the media parameters [2]. Coordinate transformations can be derived for compressing, expanding, or bending space, enabling designs of invisibility cloaks, field concentrators, perfect lenses, beam shifters, etc. Realization of these devices, however, depends on the possibility to create transformation media with prescribed properties [3], which include highly anisotropic dispersion of material parameters, as well as parameters providing wave propagation with superluminal phase velocities. Therefore, metamaterials (MMs), which were expected to exhibit most versatile properties, were considered as first candidates for realizing TO-based devices [4,5]. Application of conventional MMs composed of spilt ring resonators and cut wires, however, met serious challenges, such as increased losses in metal elements at higher frequencies, extremely narrow frequency band of operation, interresonator coupling, and the need to provide homogenization. In order to decrease losses, dielectric MMs, instead of metal ones, were used for developing invisibility cloaks for infrared range [6,7]. However, resolving all of the above listed problems would require substituting MMs in the cloak media by alternative materials. Photonic crystals (PhCs) could be considered as promising substitutes [8].

In [9] it was shown that the cloak medium, formed from fragments of 2D photonic crystals composed of dielectric rods, supported wave propagation around metal cylinder with superluminal phase velocity that provided wave-front restoration beyond the target. These results have demonstrated the perspectives of $\mathrm{PhC}$ applications in transformation media, although used in [9] crystals with square lattices could not provide TO-requested asymmetry of spatial index dispersion. It is worth noting that one complication with building a cloak from PhCs was that the number of periods required to form the band structures in $\mathrm{PhC}$ fragments affected the minimum volume of the cloak. In addition, boundaries between $\mathrm{PhC}$ fragments in the cloak medium could contribute to reflections. However, as it was shown in 
[9], the problems of both reasonable cloak thickness and excessive reflections could be solved by properly designing the cloak.

In this work, we, first, investigate the roles of spatial dispersions prescribed for orthogonal index components on the performance of cylindrical invisibility cloaks that allows for relating successful operation of PhCs-based cloak in [9] to the phenomenon of self-collimation (Section 2). Then we explore the opportunity to realize TO-requested asymmetry of dispersion laws for orthogonal index components by forming the cloak medium from anisotropic 2D $\mathrm{PhCs}$ with rectangular lattices of dielectric rods. We show that, although TO-prescribed index dispersion asymmetry could be qualitatively achieved in these $\mathrm{PhCs}$, providing the exact TO-prescribed index values by varying parameters of rod arrays in practically acceptable ranges presents a serious challenge (Sections 3 and 4). To solve the problem, we propose reduced prescriptions for spatial dispersion of radial component of index, which are compatible with capabilities of PhCs. We demonstrate that following these prescriptions does not cause significant changes in the cloak performance compared to performance of the cloak medium with parameters satisfying full TO prescriptions (Section 5). The results of full-wave field simulations presented in this work were obtained by using COMSOL Multiphysics software package, while dispersion diagrams for PhCs were calculated by using last versions of MPB software developed at MIT [10].

\section{TO-based prescriptions for orthogonal index components and functions of these components in the cylindrical cloak medium}

TO-prescriptions for the effective parameters, permittivity and permeability, of a cylindrical cloak medium have been originally proposed in [1] and later modified in [11], to avoid impedance mismatch at the outer boundary of the cloak. In order to form the cloak medium from PhCs instead of MMs, TO-prescriptions need to be re-formulated for radial and azimuthal index components of refractive index [8]. Following our earlier work [9], here we consider prescriptions to be defined by a coordinate transformation, which shrinks infinitely long cylindrical space, represented in cylindrical coordinate system by radius $r^{\prime}$, into an annular cylindrical space, represented by radius $r$ :

$$
r=f\left(r^{\prime}\right)=\left(1-\frac{R_{\text {in }}}{R_{\text {out }}}+\frac{R_{\text {in }}}{R_{\text {out }}^{2}}\left(r^{\prime}-R_{\text {out }}\right)\right) r^{\prime}+R_{\text {in }} ; \theta=\theta^{\prime} ; z=z^{\prime}
$$

where $0 \leq r^{\prime} \leq R_{\text {out }}$ and $R_{\text {in }} \leq r \leq R_{\text {out }}$, while the values $R_{\text {in }}$ and $R_{\text {out }}$ are, respectively, the inner and the outer radii of the cloaking shell (Fig. 1 (a)). At TM polarization of incident waves (magnetic field directed along $z$-axis of the cylindrical cloak), prescriptions for effective permeability of the cloak medium could be reduced to $\mu_{z}=1$ [5], while for the components of effective permittivity in E-field plane (normal to the cloak axis) following expressions could be obtained following [11]:

$$
\varepsilon_{r}=\left(\frac{r^{\prime}}{r}\right)^{2} ; \varepsilon_{\theta}=\left(\frac{d f\left(r^{\prime}\right)}{d r^{\prime}}\right)^{-2}
$$

Expressions (2) could be then used to derive index components for wave propagation in azimuthal and radial directions (Fig. 1 (a)) by utilizing dependencies of these components on effective parameters of the medium: $n_{r}=\sqrt{\varepsilon_{\theta} \mu_{z}}$ and $n_{\theta}=\sqrt{\varepsilon_{r} \mu_{z}}$. Accordingly, we obtain:

$$
\begin{gathered}
n_{r}=\left(1-\frac{2 R_{\mathrm{in}}}{R_{\text {out }}}+\frac{2 R_{\mathrm{in}} r^{\prime}}{R_{\text {out }}^{2}}\right)^{-1} \\
n_{\theta}=\frac{r^{\prime}}{r}
\end{gathered}
$$

where $r^{\prime}$ could be found from equation (1) as following: 
Equations (3-5) could be used for calculating prescribed by TO spatial dispersions of index components. Curves A and F in Fig. 1 (b) present these dispersions for a cloaking shell having $\frac{R_{\text {out }}}{R_{\text {in }}}=3.5$, which is hiding the target with the diameter of $74 \mathrm{~mm}$. As seen in the figure, TO prescribes ascending dispersion from inner to outer cloak layers (curve A) for azimuthal index component and descending dispersion (curve F) for radial index component. In addition, azimuthal index component should have values less than 1 within the entire cloak, i.e. these values should support wave propagation with superluminal phase velocities, while radial index component, just opposite, should be higher than 1, i.e. its values should support refraction phenomena, turning waves around the cloak axis.

Considering the described above functions of two index components caused by their specific spatial dispersions, it could be suggested that employing PhCs with square lattices in the cloak medium, as in [9], should exclude obtaining the cloaking effect. In fact, providing superluminal index values in azimuthal direction in PhCs with square lattices had to introduce similar values for indices in radial direction. Fig. 2 (a) presents the wave pattern simulated for the cloak model with identical dispersions of index components in two directions (corresponding to curve A in Fig. 1 (b)). The frequency of $11.4 \mathrm{GHz}$ was taken arbitrary, since index values in the model were taken to be frequencyindependent. As seen in the figure, a typical shadow behind the metal target is observed instead of the cloaking effect. Wave-patterns presented in Figs. from 2 (b) to 2 (f) correspond to the cloak models with dispersions for radial index component changing from curve $\mathrm{B}$ to curve $\mathrm{F}$ in Fig. 1 (b), respectively, while dispersion for azimuthal index component was kept as curve A in Fig. 1 (b). It can be seen that significant decrease of the shadow occurred only at radial index values exceeding 1 (and essentially exceeding 1 near the target) with radial dispersion approaching curve F in Fig. 1 (b). It is obvious from the obtained results that the media with dispersions following curve A for both index components should not provide the cloaking effect, unless any other physical mechanism, instead of refraction, can support wave turning around the target.

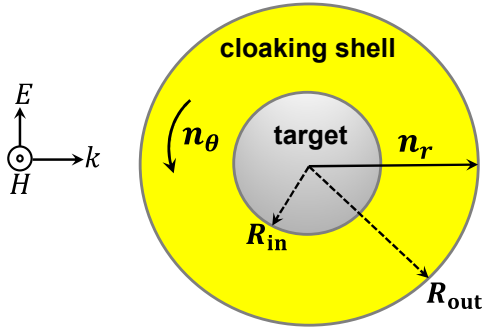

(a)

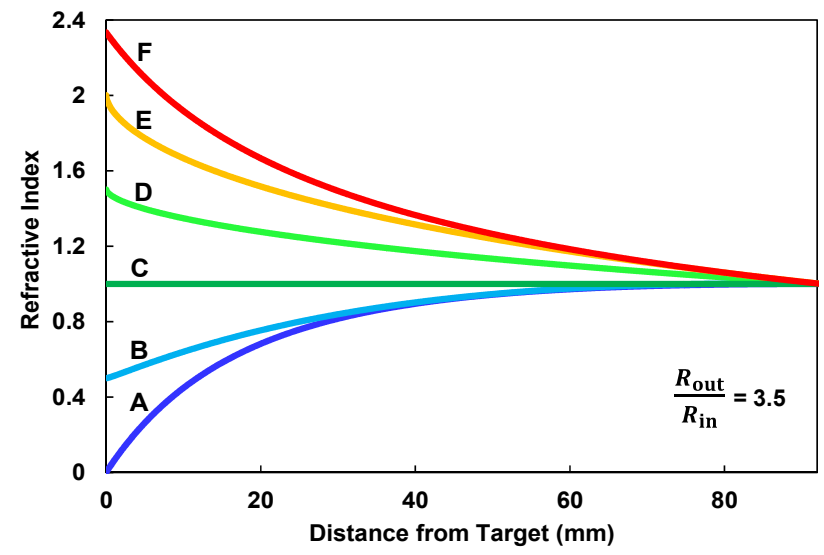

(b)

Figure 1. (a) Cross-section of cylindrical cloak and (b) various spatial distributions of index within cloaking shell with $\frac{R_{\mathrm{out}}}{R_{\mathrm{in}}}=3.5$.

This mechanism could be related to the known in PhCs phenomenon of self-collimation. According to [12], due to this phenomenon, PhCs could support wave propagation along crystallographic axes, even if they are bent. Indeed, Fig. 3 taken from a row of images of wave-patterns in [9] just demonstrates self-collimated wave movement in outer $\mathrm{PhC}$ layers of the cloak, while beyond the cloak, a shadow similar to that presented in Fig. 2 (a) can be clearly seen. This observation opens up an opportunity for employing self-collimation in PhCs for providing TO-requested functionalities of the cloak medium, when prescriptions for material properties could not be realized in full. 


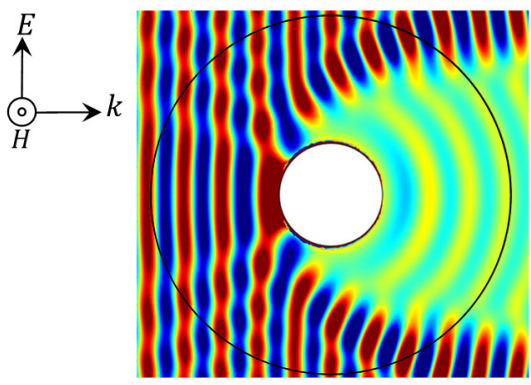

(a)

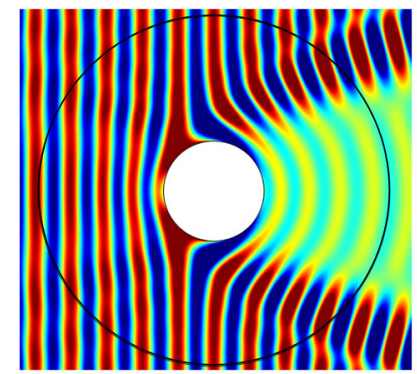

(d)

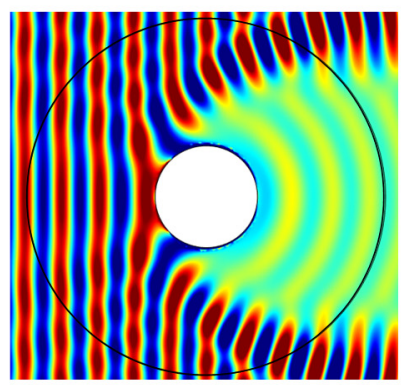

(b)

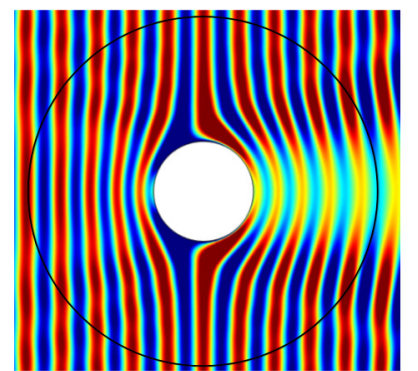

(e)

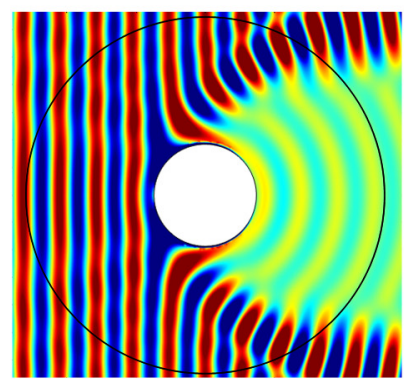

(c)

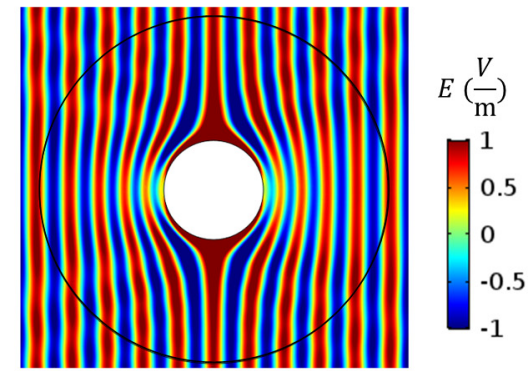

(f)

Figure 2. Simulated wave patterns at $11.4 \mathrm{GHz}$ for the cloak with $\frac{R_{\text {out }}}{R_{\mathrm{in}}}=3.5$ for (a) curve A in Fig. 1 (b) as radial and azimuthal index dispersions; (b), (c), (d), (e), and (f): curve $\mathrm{A}$ as azimuthal index dispersion, while radial index dispersion is following curves B, C, D, E, and F in Fig. 1 (b), respectively.

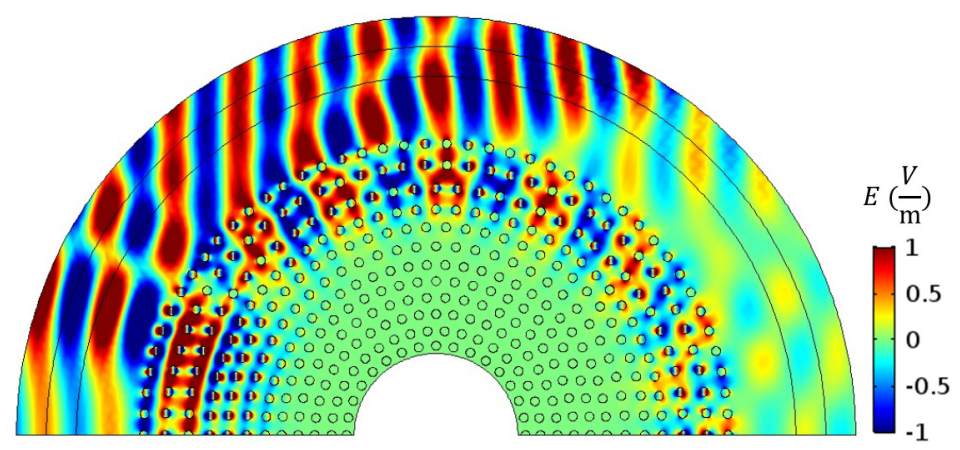

Figure 3. Wave-pattern for the cloak in [9] formed from square-lattice PhCs demonstrates the effect of self-collimation at 13.6 GHz.

\section{Achieving anisotropic index dispersion in PhC-based cloak media}

As it was shown in Section 2, if self-collimation is not realized in the cloak medium formed by PhCs with square lattices, cloaking effect could not be observed in such medium, since TO-prescribed difference between spatial dispersions for azimuthal and radial index components could not be provided. However, according to [13, 14], difference between index component for wave propagation in two orthogonal directions could be achieved in PhCs with rectangular lattices. Therefore, we explored an opportunity to employ such $\mathrm{PhCs}$ in the cloak medium for obtaining desired difference of spatial dispersions of $n_{r}$ and $n_{\theta}$.

In [9] it was demonstrated that changing the lattice constant of $\mathrm{PhC}$ caused shifting of the $2^{\text {nd }}$ transmission band in its dispersion diagram along the frequency axis. This also caused respective shift of the extracted from the dispersion diagram frequency dependence of index values. It was also proven in [9] that the $2^{\text {nd }}$ branch of array dispersion diagram 
supplied index values in the range from zero to higher than 1 . Therefore, it was possible to determine a set of array lattice constants providing at some frequency a collection of index values representing the spatial dispersion law depicted by curve A in Fig. 1 (b). The requested dispersion law was then realized in the cloak medium by building it from fragments of $\mathrm{PhCs}$ with respective lattice constants.

In order to investigate, how the difference in lattice constants along two directions would affect the dependencies of index values on frequency, we first calculated dispersion diagrams for PhCs with rectangular lattices at TM wave incidence along either one of two crystallographic directions, which we denoted as $x$ and $y$. Fig. 4 (a) presents $2^{\text {nd }}$ branches of dispersion diagrams within $\Gamma$-X range of wave-vectors for arrays with relative rod permittivity $\varepsilon=35$ and $R=1.5 \mathrm{~mm}$ (same rod parameters as in [9]), with the same lattice constant $a_{x}$ and with various lattice constants $a_{y}$. As seen in the figure, difference between $a_{y}$ and $a_{x}$ results in changes of dispersion diagrams, i.e. in anisotropy of array properties. From dispersion diagrams, the values of $x$ - and $y$-components of index were calculated by using wellknown relation given in [15]:

$$
n_{\mathrm{eff}}=\operatorname{sgn}\left(v_{g} \cdot k\right)\left(\frac{c}{\omega}|k|\right)
$$

where $c$ is the speed of light in free space and $v_{g}=\frac{d \omega}{d k}$ is the group velocity, while $k$ and $\omega$ are wave vector and angular frequency, respectively. Equation 6 characterizes the dominant refractive index. As discussed in $[9,15,16]$, this approach provides the effective values of phase refractive indices in the second transmission bands of 2D PhCs. In [9], this approach has been verified by comparing the indices obtained from the dispersion diagrams of 2D rod arrays (at various rod permittivities) with the results of the index retrieval procedure.

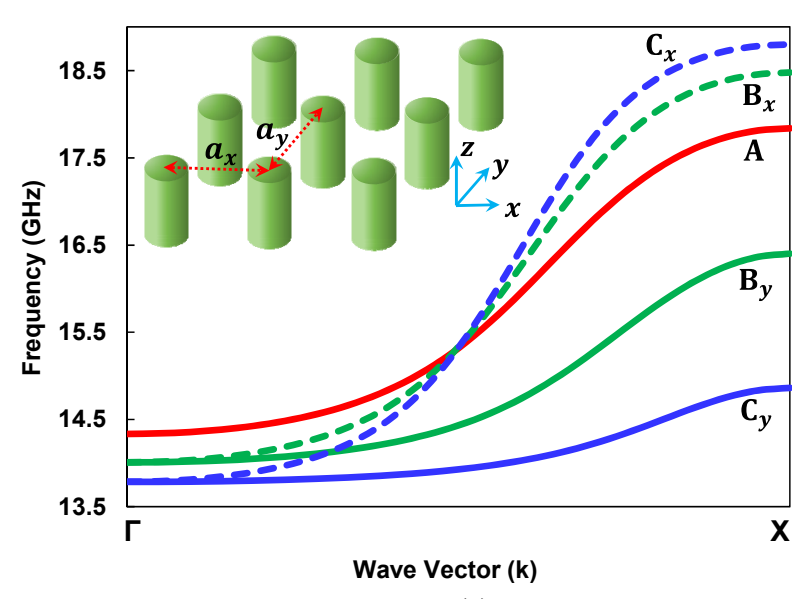

(a)

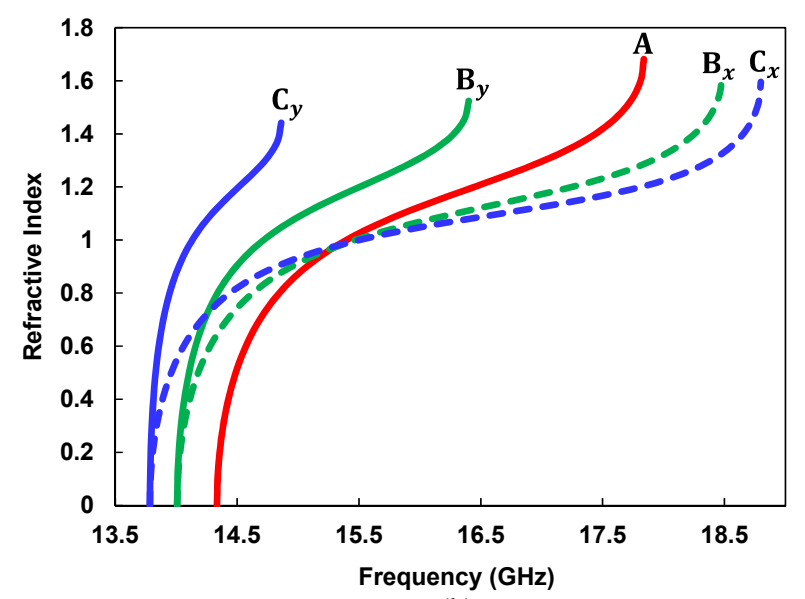

(b)

Figure 4. (a) $2^{\text {nd }}$ branch of dispersion diagrams for rod arrays when incident TM waves are propagating in either $x$ or $y$ directions: $a_{x}$ is fixed at $5 \mathrm{~mm}$ while $a_{y}$ for curves $\mathrm{A}, \mathrm{B}$, and C is $5 \mathrm{~mm}, 6 \mathrm{~mm}$, and $7 \mathrm{~mm}$, respectively. (b) Dependencies of refractive index components $n_{x}$ and $n_{y}$ on frequency calculated using dispersion diagrams in (a).

Fig. 4 (b) presents frequency dependencies of $n_{x}$ and $n_{y}$ found by using respective diagrams in Fig. 4 (a). As seen in Fig. 4 (b), at $a_{x}=a_{y}$ frequency dependencies of $n_{x}$ and $n_{y}$ coincide (curve A). At $a_{y}>a_{x}$ dependencies for $n_{x}$ and $n_{y}$ shift and split, so that dependencies for $n_{y}$ get steeper, while dependencies for $n_{x}$ become less steep and, therefore, cross the curve A. Shifting of the spectra of $n_{y}$ and $n_{x}$ reflects lowering of the edge of the $2^{\text {nd }}$ transmission band, which is well seen in Fig. 4 (a). It could be also noticed in Fig. 4 (b) that at increased values of $a_{y}, n_{y}$ values become significantly exceeding 1 at such frequencies, at which the values of slower growing $n_{x}$ still remain below 1 . Such difference between $n_{y}$ and $n_{x}$ in arrays with rectangular lattices seems to point out at an opportunity of realizing desired anisotropy of TO prescriptions for index components in two orthogonal directions.

Further investigations, however, have shown that obtaining thus high values of $n_{y}$, as defined by curve $\mathrm{F}$ in Fig. 1 (b), is challenging for PhCs, since having much bigger $a_{y}$ compared to $a_{x}$ does not allow for achieving much higher 
index values than those presented in Fig. 4 (b). Instead, it leads, first, to extinction of the $2^{\text {nd }}$ branch and then, to switching of the sign of $n_{y}$ from positive to negative, which is not suitable for controlling wave propagation in the cloak. Thus, there are limits for increasing $a_{y}$ at any chosen $a_{x}$. For example, at $a_{x}=5 \mathrm{~mm}, a_{y}$ should not exceed 8 $\mathrm{mm}$ to avoid negative index values. At $a_{y}<8 \mathrm{~mm}$ and used in [9] rod parameters ( $\varepsilon=35$ and $R=1.5 \mathrm{~mm}$ ), $n_{y}$ values bigger than 1.5 cannot be achieved, while they should be close to 2.4 near the target, according to curve F in Fig. 1 (b) for the cloak with $\frac{R_{\text {out }}}{R_{\text {in }}}=3.5$. Taking this into account, we looked for a set of arrays with rectangular lattices and with the same, as in [9], rod parameters, which could be used for providing, at some frequency, best fit of index values to dispersion curves A and F in Fig. 1 (b). We considered a set of four arrays, the fragments of which could be used for assembling the cloak, for which prescribed dispersion curves for index components would be represented by stepfunctions consisting of four steps. Fig. 5 shows extracted from dispersion diagrams frequency dependencies of $n_{y}$ and $n_{x}$ values for such four arrays, having different combination of lattice constants $a_{x}$ and $a_{y}$. The presented data demonstrate that combining fragments of these arrays in the cloak medium can provide descending spatial dispersion for radial index component and ascending spatial dispersion for azimuthal component at the operation frequency of $13.73 \mathrm{GHz}$, although the prescribed maximal value of 2.4 for $n_{y}$ cannot be achieved. This result pushed us to searching for opportunities to decrease maximal $n_{r}$ values, prescribed by TO, by varying cloak dimensions and to increase $n_{y}$ values, achievable in rod arrays with rectangular lattices, by modifying rod radius and permittivity.

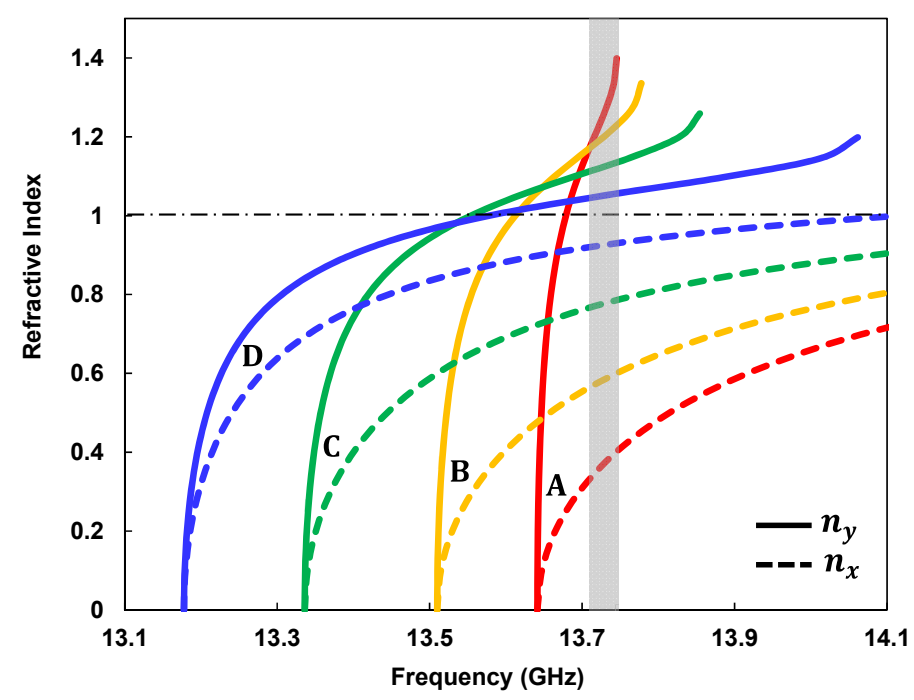

Figure 5. Frequency dependencies of directional index values in the $2^{\text {nd }}$ transmission bands for four arrays with rod parameters: $\varepsilon$ $=35$ and $R=1.5 \mathrm{~mm}$, and with rectangular lattices; $a_{x}$ and $a_{y}$ values (in $\mathrm{mm}$ ) for the curve couples A, B, C, and D, are, respectively: $(5 ; 7.8),(5.5 ; 8.15),(6.5 ; 8.6)$, and $(8 ; 8.9)$.

\section{Fitting capabilities of rod arrays to TO prescriptions for index components}

From the expressions given in Section 2, it follows that requested index values strongly depend on the ratio $\frac{R_{\text {out }}}{R_{\text {in }}}$. Fig. 6 demonstrates that by increasing this ratio, prescribed radial index values near the target can be essentially reduced, while azimuthal index values are just slightly affected. Thus, as seen in Fig. 6, capabilities of rod arrays would become sufficient for satisfying TO prescriptions at $\frac{R_{\text {out }}}{R_{\text {in }}}>4.6$. This ratio, however, characterizes, in fact, the thickness of the cloak, so that increasing this ratio means significant increase of the cloak thickness at fixed size of the target. In particular, changing the ratio from 3.5 to 4.6 corresponds to $44 \%$ increase of the cloak thickness, which is, obviously, not desirable for practical applications. 
Another approach to satisfying TO prescriptions for the cloak medium formed from rod arrays could be seen in manipulating parameters of rods. As demonstrated in Fig. 7 (a), increasing rod radius from $1.5 \mathrm{~mm}$ up to $1.9 \mathrm{~mm}$ provided an increase of highest achievable $n_{y}$ value in PhCs up to 1.8, which corresponded to TO requirements for cloak thickness defined by the ratio $\frac{R_{\text {out }}}{R_{\text {in }}}=4.6$, although it was still below the requirements for smaller cloak thickness, when $\frac{R_{\text {out }}}{R_{\text {in }}}=3.5$. The effect of another parameter, i.e. rod permittivity, on achievable index values in rod arrays, is demonstrated in Fig. 7 (b). As seen in the figure, changing rod permittivity did not influence effectively the highest values of $n_{y}$, however, it allowed for decreasing the asymmetry of array responses along $x$ and $y$ directions and made frequency dependences of both index components $n_{y}$ and $n_{x}$ less steep that opened up a window for playing with radii of rods and/or lattice parameters of arrays with the aim of providing higher $n_{y}$ values. Fig. 7 (c) shows the effect of increasing rod radius in arrays with slightly higher rod permittivity than that used in Fig. 7 (a). From comparing Figs. 7 (a) and (c), it can be seen that an increase of rod permittivity from 35 to 39 allows for approaching $n_{y}$ value of 2.0, while at permittivity of 35 (as in [9]) it was impossible to reach values of $n_{y}$ exceeding 1.8. Although performed studies have shown that combined variations of cloak thickness, rod permittivity, and rod radii allow for approaching TO-requested prescriptions for index components in the cloak medium, it would be desirable to search for such reduced prescriptions, which could make practical implementation of PhC-based cloak much more feasible. 


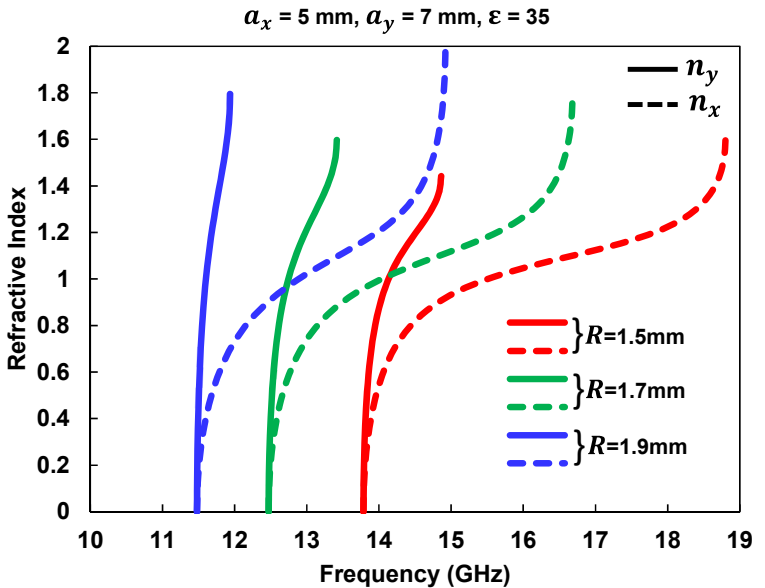

(a)

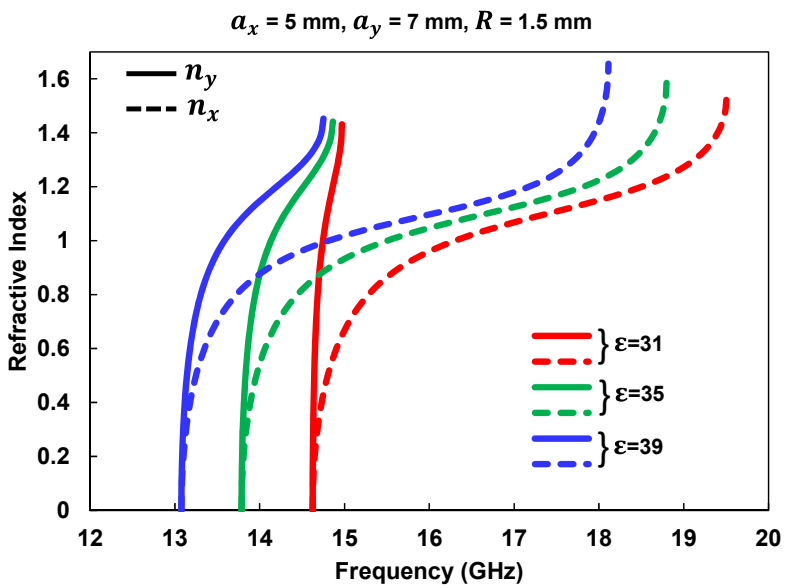

(b)

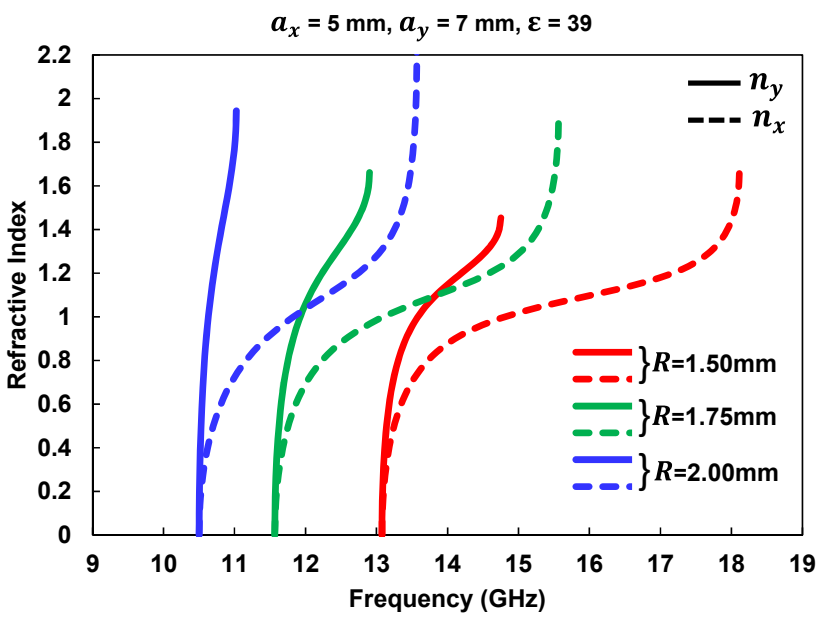

(c)

Figure 7. Frequency dependencies of directional index values in $2^{\text {nd }}$ transmission bands of rectangular arrays with $a_{x}=5 \mathrm{~mm}$ and $a_{y}=7 \mathrm{~mm}$ for various values of: (a) rod radius at $\varepsilon=35$, (b) rod permittivity at $R=1.5 \mathrm{~mm}$, and (c) rod radius at $\varepsilon=39$.

\section{Reduced prescriptions for spatial dispersion of index components in the cloak medium}

Here we consider an opportunity to replace TO-based prescriptions by reduced spatial dispersion law for radial index component. Our approach is based on understanding of the main function of radial index component in governing wave propagation in the cloak. As it follows from Section 2, the role of radial indices can be described as turning waves around the target. To accomplish this task, TO prescriptions for the radial index component demand very high index values near the target and steep decrease of these values further from the target. In order to restrict TO demands by physically achievable in rod arrays index values, we were looking for reduced dispersion laws for $n_{r}$, which would still be able to accomplish the function of turning waves around the target. In particular, we looked for dispersion laws with decreased against TO prescriptions $n_{r}$ values near the target and increased, for compensating this decrease, $n_{r}$ values in outer cloak layers, as shown by solid red curve in Fig. 8. To choose appropriate analytical expression describing $n_{r}$ dispersion (solid red curve in Fig. 8), various functions were tested. Basic criteria, employed for the choice, were restoration of the flat wave front behind the cloak and maximal decrease of the total scattering crosswidth of the cloaked target versus that of the bare target. Based on these criteria, the expression given below, which is controlled by three parameters, was used as the reduced prescription for $n_{r}$ dispersion:

$$
n_{r}=\alpha^{1-\left(\frac{\frac{r}{R_{\mathrm{in}}}-1}{\beta}\right)^{\gamma}} ; \quad R_{\text {in }} \leq r \leq R_{\text {out }}
$$


where $\alpha, \beta$, and $\gamma$ are, respectively, the parameter controlling the value of $n_{r}$ at inner boundary of the cloak, the parameter controlling the lowest level of $n_{r}$ (at outer boundary of the cloak), and the parameter managing the steepness of radial index dispersion. Expression (7) has been obtained at transforming the well-known decaying function $n_{r}=\alpha^{1-x}$, where $0<x<1$ is the distance from some origin. Since, in our case, the distance of interest was defined by the distance between $R_{\text {in }}$ and $R_{\text {out }}$, we, first, replaced $x$ by expression $\frac{r}{R_{\mathrm{in}}}-1$, which became equal to zero at $R=R_{\text {in }}$ and, thus, provided $n_{r}=\alpha$ at the inner boundary of the cloak. Then we introduced additional complication in the definition of $x$ as: $x=\frac{\frac{r}{R_{\text {in }}}-1}{\beta}$, which led to $x=1$ at the outer cloak boundary and, respectively, to $n_{r}=1$, if $\beta=\frac{R_{\text {out }}}{R_{\text {in }}}-1$. Taking the value of $\beta$ bigger than $\frac{R_{\text {out }}}{R_{\text {in }}}-1$ allowed for requesting the value of $n_{r}$ at the outer boundary of the cloak to be bigger than 1 up to desired level. Introducing coefficient $\gamma$ in the expression for $n_{r}$ provided additional option for manipulating the dispersion law at the search for the law providing better fit to the described above criteria.

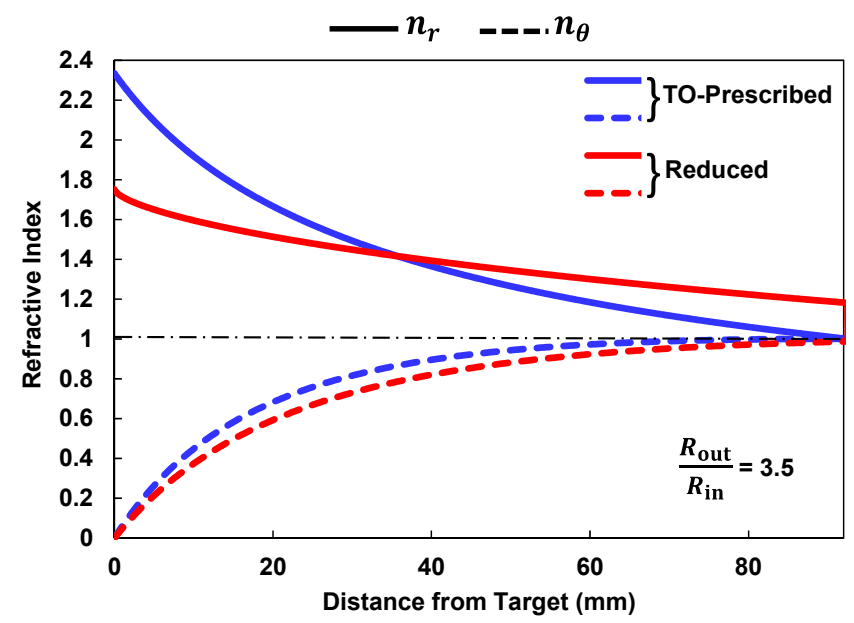

Figure 8. TO-prescribed and reduced (based on Eq. 7 at $\alpha=1.75, \beta=4.3$, and $\gamma=0.65$ ) index dispersions for cloak with $\frac{R_{\text {out }}}{R_{\text {in }}}=$ 3.5 .

Proposed approach can be illustrated by finding an appropriate reduced prescription for the cloak with $\frac{R_{\text {out }}}{R_{\text {in }}}=3.5$. First, we determined the values of parameters $\alpha, \beta$, and $\gamma$, at which the dispersion law, prescribed by expression (7), would fit TO-prescribed dependence presented by curve F in Fig. 1 (b). Red curve in Fig. 9 (a) illustrates the result of fitting. Then, by reducing the value of $\alpha$, as shown in Fig. 9 (a), the highest value of $n_{r}$ near the target has been lowered down to less than 1.8, which was attainable in rod array with rectangular lattice, rod radius $R=1.9 \mathrm{~mm}$, and $\varepsilon=35$ (blue curve in Fig. 9 (a)). Then, to increase $n_{r}$ values in outer layers of the cloak with the aim to compensate for weaker refraction caused by reduced values of $n_{r}$ near the target, we employed higher than 2.5 values of $\beta$ in expression (7) and have chosen $\beta$ to be equal 4.3 to obtain $n_{r} \simeq 1.25$ at the outer boundary of the cloak (blue curve in Fig. 9 (b)). Finally, we varied $\gamma$ around the value of 0.7 used at fitting the dispersion law prescribed by expression (7) to TO-prescribed law given by curve F in Fig. 1 (b) (see Fig. 9 (c)). 


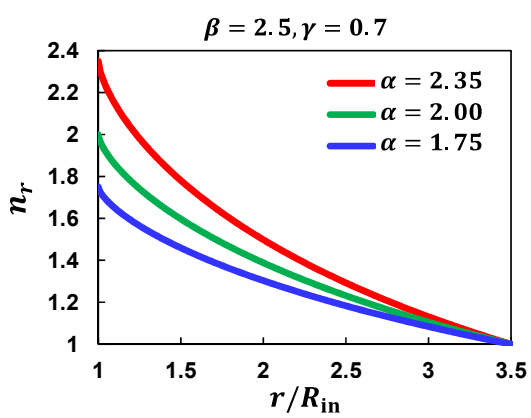

(a)

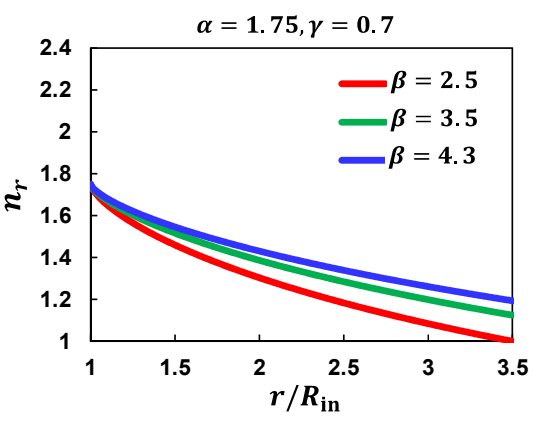

(b)

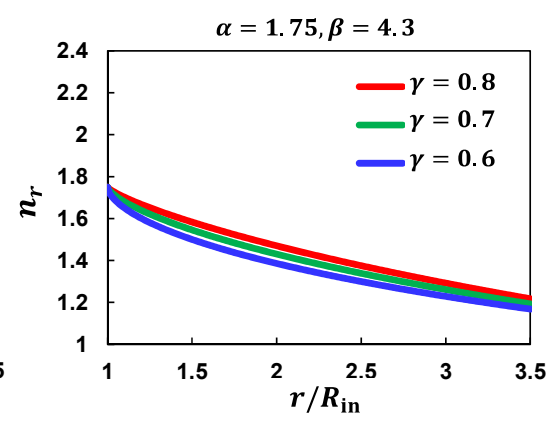

(c)

Figure 9. Calculated radial index dispersions according to expression (7) for various $\alpha, \beta$, and $\gamma$ at $\frac{R_{\text {out }}}{R_{\mathrm{in}}}=3.5$.

Wave propagation through cloaks with media obeying different dispersion laws for $n_{r}$, was simulated and compared to wave propagation through the cloak with TO-prescribed dispersion (red curve in Fig. 9 (a)). In addition, COMSOL software was used to calculate total scattering cross-widths for the cloaks under study, following [17]. The best performance of the cloak employing reduced prescriptions, which was quite comparable to the performance of the cloak based on TO prescriptions at $\frac{R_{\text {out }}}{R_{\text {in }}}=3.5$, was demonstrated for the values of parameters $\alpha, \beta$, and $\gamma$ equal to $1.75,4.3$ and 0.65 , respectively. Fig. 10 demonstrates that the target (metal cylinder) covered by the cloak having reduced index dispersion, causes only slightly higher scattering compared to scattering caused by the target covered with TO-prescribed cloak, both being much lower than scattering caused by bare target.

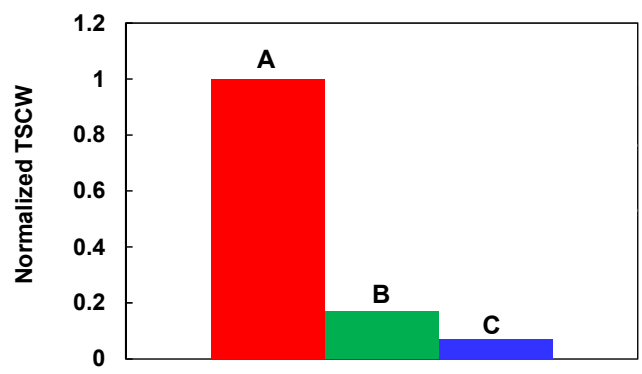

Figure 10. Calculated total scatterings cross-width of bare target (A), of target covered by cloak with reduced index dispersion (B), and of target covered by cloak with TO-prescribed index dispersion (C).

As seen in Fig. 11, simulated field patterns for wave propagation through metal cylinders concealed by cloaks with TO-prescribed and reduced index dispersions, look almost identical. It could be concluded, that the main function of TO-prescribed spatial dispersion for radial index component in the cloak medium, which can be described as turning the paths of incident waves around the target with minimal reflection or scattering, could be achieved at reduced prescriptions, if they provide compensation of weaker refraction near the target by enhanced refraction in outer cloak layers. The main advantage of reducing the maximum value of $n_{r}$ near the target is an opportunity to realize properly performing cloak by using dielectric PhCs with rectangular lattices. It is worth mentioning here that earlier in [3], where the cloak medium was composed of MMs, TO requests to spatial dispersions of material parameters have also been reduced. Original $\varepsilon_{Z}$ and $\mu_{\theta}$ distributions in [3] were approximated by constant values, and $\mu_{r}$ was the only radially varied parameter that made the realization of the cloak essentially easier. However, this cloak did not provide appropriate wave-front reconstruction beyond the target and demonstrated a non-negligible shadow, characteristic for improper realization of refraction demands. Similar, as in [3], approach was used at building the cloak from dielectricmetal MMs in [18], where $\mu_{z}$ and $\varepsilon_{\theta}$ were reduced to constant values, while $\varepsilon_{r}$ was changing from 0 at inner boundary of the cloak to 1 at outer boundary. Similar to [3], power flow beyond the cloak with reduced material parameters was found to be essentially lower than that in the case of the cloak with full parameters prescribed by TO. 


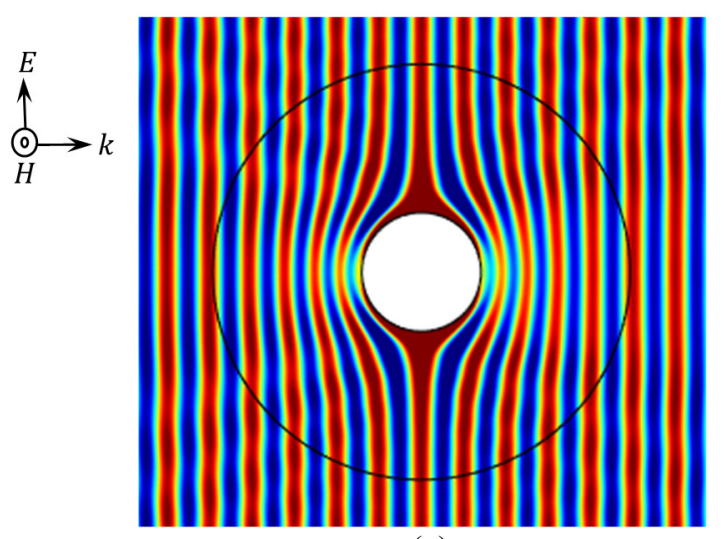

(a)

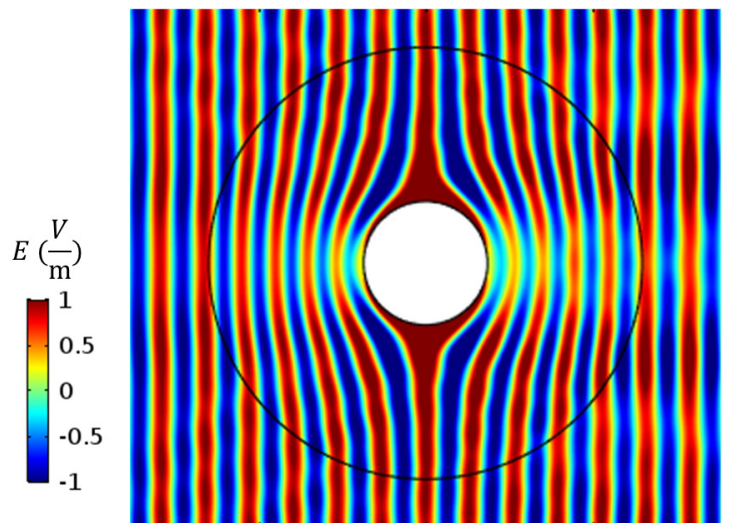

(b)

Figure 11. Simulated wave-patterns at an arbitrary frequency of $11.4 \mathrm{GHz}$, at TM wave incidence from left on cloaked targets with $\frac{R_{\text {out }}}{R_{\text {in }}}=3.5$ : (a) at TO-prescribed index dispersion, (b) at reduced dispersion of radial index component.

\section{Conclusion}

We have shown that for obtaining proper cloaking effect, cloak medium, formed from PhCs, should have its radial index component changing from values exceeding 1 near the target down to 1 at the outer boundary of the cloak, in addition to ascending dispersion for azimuthal index component, unless waves are guided around the target due to self-collimation phenomenon mimicking refraction effects. It is demonstrated that two types of spatial dispersions prescribed for index components of cylindrical cloaks can be realized in the media built from 2D PhCs with rectangular lattices of dielectric rods. Such crystals were shown capable of supporting, in the $2^{\text {nd }}$ transmission bands, index values ranging from zero to those significantly exceeding 1 . Increasing $\mathrm{PhC}$ 's lattice constants allowed for shifting their $2^{\text {nd }}$ bands in dispersion diagrams down to lower frequencies thus providing spatial dispersion of indices in the cloak composed of $\mathrm{PhCs}$ fragments with different lattice constants. However, maximal values of radial indices, which could be obtained in PhCs at increasing lattice asymmetry, appeared to be less than those prescribed by TO because of restrictions imposed on crystal asymmetry by extinction of the $2^{\text {nd }}$ transmission band. Changes of the cloak thickness and rod parameters were found incapable of completely closing the gap between TO prescriptions and modalities of used PhCs. To solve this problem, we proposed reduced prescriptions for radial index dispersion. Reduced dispersion law suggested employing essentially smaller values of radial index near the target, than those requested by TO, so that these values would be achievable by using crystal asymmetry. To compensate for weaker refraction in inner layers, we proposed to provide higher than requested by TO index values in outer layers of the cloak. Conducted simulations of the cloaks with reduced spatial dispersions of radial indices have shown that these cloaks perform similarly to TOfollowing cloaks, i.e. support wave front restoration beyond the target and drastic decrease of the total scattering crosswidth by the cloak. These results make feasible practical realization of invisibility cloak by using PhCs with rectangular lattices.

\section{Acknowledgments}

This work was supported by the National Science Foundation under Award ECCS-1709991.

\section{References:}

[1] J. B. Pendry, D. Schurig, and D. R. Smith, 'Controlling Electromagnetic Fields,' Science, Vol 312, 23 , June 2006. [2] Y. Nathan, B. Kundtz, D. R. Smith, and J. B. Pendry, 'Electromagnetic Design with Transformation Optics,' Proceedings of the IEEE, Vol. 99, No. 10, October 2011.

[3] S. A. Cummer, B.-I. Popa, D. Schurig, D. R. Smith, and J. Pendry, 'Full-Wave Simulations of Electromagnetic Cloaking Structures,' Physical Review E 74, 036621, 2006.

[4] D. Schurig, J. J. Mock, B. J. Justice, S. A. Cummer, J. B. Pendry, A. F. Starr, and D. R. Smith, 'Metamaterial Electromagnetic Cloak at Microwave Frequencies‘ Science, Vol 314, 10, Nov. 2006. 
[5] W. Cai, U. K. Chettiar, A. V. Kildishev, and V. M. Shalaev, 'Optical Cloaking with Metamaterials,' Nature Photonics, Vol 1, April 2007.

[6] D. P. Gaillot, C. Croënne, F. Zhang, and D. Lippens 'Transformation Optics for the Full Dielectric Electromagnetic Cloak and Metal-Dielectric Planar Hyperlens,' New Journal of Physics, Vol. 10, 115039, Nov. 2008.

[7] E. Semouchkina, D. H. Werner, G. B. Semouchkin, and C. Pantano, 'An Infrared Invisibility Cloak Composed of Glass,' Applied Physics Letters, 96, 233503, 2010.

[8] Y. A. Urzhumov and D. R. Smith, 'Transformation Optics with Photonic Band Gap Media' Physical Review Letters, 105, 163901, 2010.

[9] E. Semouchkina, R. Duan, N. P. Gandji, S. Jamilan, G. Semouchkin, and R. Pandey, 'Superluminal Media Formed by Photonic Crystals for Transformation Optics-Based Invisibility Cloaks,' Journal of Optics, 18, 044007, 2016.

[10] http://ab-initio.mit.edu/wiki/index.php/MIT_Photonic_Bands

[11] W. Cai, U. K. Chettiar, A. V. Kildishev, V. M. Shalaev, and G. W. Milton, 'Nonmagnetic Cloak with Minimized Scattering,' Applied Physics Letters, 91, 111105, 2007.

[12] R. C. Rumpf, J. Pazos, C. R. Garcia, L. Ochoa, and R. Wicker, '3D Printed Lattices with Spatially Variant SelfCollimation,' Progress In Electromagnetics Research, Vol. 139, 2013.

[13] Y. Takayama and W. Klaus, 'Refractive Behavior of 2D Square Lattice Photonic Crystals Determined by Reducing the Symmetry of the Unit Cell,' Japanese Journal of Applied Physics, Vol. 41, No. 11A, pp. 6375-6379, Nov. 2002.

[14] L. Peng, L. Ran, and N. A. Mortensen, 'Achieving Anisotropy in Metamaterials Made of Dielectric Cylindrical Rods,' Applied Physics Letters, 96, 241108, 2010.

[15] P.V. Parimi, W.T. Lu, P. Vodo, J. Sokoloff, J. S. Derov, and S. Sridhar, 'Negative Refraction and Left-Handed Electromagnetism in Microwave Photonic Crystals,' Physical Review Letters, Vol 92, No 12, p. 127401, March 2004.

[16] M. Notomi, 'Theory of Light Propagation in Strongly Modulated Photonic Crystals: Refractionlike Behavior in the Vicinity of the Photonic Band Gap,' Physical Review B, Vol. 62, No. 16, Oct. 2000.

[17] https://www.comsol.com/model/radar-cross-section-8613.

[18] D. Diedrich, A. Rottler, D. Heitmann, and S. Mendach, 'Metal-Dielectric Metamaterials for TransformationOptics and Gradient-Index Devices in the Visible Regime,' New Journal of Physics, 14, 053042, 2012. 